\title{
The Ne Plus Ultra of Tree Graph Inequalities ${ }^{\star}$
}

\author{
PAUL FEDERBUSH \\ Department of Mathematics, University of Michigan, Ann Arbor, MI 48109, U.S.A.
}

(Recelved: 31 August 1989)

\begin{abstract}
With nary mention of a tree graph, we obtain a cluster expansion bound that includes and vastly generalizes bounds as obtained by extant tree graph inequalities. This includes applications to both two-body and many-body potential situations of the recently obtained 'new improved tree graph inequalities' that have led to the 'extra $1 / N$ ! factors'. We work in a formalism coupling a discrete set of boson variables, such as occurs in a lattice system in classical statistical mechanics, or in Euclidean quantum field theory. The estimates of this Letter apply to numerical factors as arising in cluster expansions, due to essentially arbitrary sequences of the basic operations: interpolation of the covariance, interpolation of the interaction, and integration by parts. This includes complicated evolutions, such as the repeated use of interpolation to decouple the same variables several times, to ensure higher connectivity for renormalization purposes, in quantum field theory.
\end{abstract}

AMS subject classifications (1980). 82A67, 81E10.

Tree graph inequalities are intimately involved in cluster expansion estimation. The earliest tree graph inequalities were developed by Glimm and Jaffe in applications to quantum field theory. But, in fact, they have important applications in simpler situations, such as in the study of the Mayer expansion in classical statistical mechanics. Perhaps the simplest cluster expansion (in the most leisurely presentation), using tree graph estimation, is given in [3], developing the Mayer expansion in a classical gas with two-body interactions. (See also [5], page 40.)

In [1], an improved method of tree graph estimation was developed. (In this paper [1], the case of two-body interactions is treated in Section 8, and the case of many-body interactions in Appendix B. But it should be noted that in cluster expansions involving many-body interactions, one can often organize the combinatoric estimates, by grouping variables together, so that one only needs the same bounds as apply in the two-body case. This was true for the problem studied in [1].) With this new tree graph estimation procedure, 'Extra $1 / N$ ! factors' are obtainable in bounds; these have been important in many applications. This estimation procedure has been further exposed in [2] and [4]. The moral of this development was that to bound contributions as arising in a cluster expansion one should group terms with the same 'interaction structure' together. Using a common estimate for the interaction structure, the sum of the integrals over interpolation parameters adds to a simple numerical weighting. (In fact equalling one!) One does not

* This work was supported in part by the National Science Foundation under grant no. PHY-87-01329. 
distinguish different histories of development of these terms, different orders of differentiation. Estimation thus pursued yielded the 'extra $1 / N$ ! factors', and was easy to accommodate to the 'sums to sups' procedure standard in cluster expansion study.

In the cluster expansion applications discussed in the last paragraph, each term in the cluster expansion is associated to a tree graph, with each vertex associated to a subset of variables, and with the vertices ordered. Each vertex arises from an expansion step in the cluster expansion, the ordering as given by the sequence of steps, the variables as introduced at the given step. The new tree graph estimation procedure of $[1,2,4]$ consists primarily of disregarding the ordering, grouping terms together associated to tree graphs with the same structure, the same variables at each vertex, but possibly different orders of vertices - such terms are said to have the same 'interaction structure'. In the present Letter, we will deal with cluster expansion terms not necessarily associated to tree graph structures, but to diagrams with higher connectivity structure.

In this Letter, we treat an expectation as given in (1), below, and consider it expanded as a sum of terms, in (8), below. The expansion is developed employing the unit operations of interpolation of the interaction, interpolation of the covariance, and integration by parts (the first two operations occur combined). We assume familiarity with these general operations, and explicit steps in minimal detail basically to establish notation. In developing a connected kernel in a cluster expansion, or a polymer, these operations are inductively performed according to carefully prescribed rules. The usual tree graph estimation procedure is intimately entangled with these expansion rules. Our estimate, Equation (16) below, holds for any evolution of the unit operations, essentially arbitrary interpolations, and is not naturally associated to any tree graph estimation. Such more complicated evolutions are important in the phase cell expansions of quantum field theories [1]. One may, for example, use interpolation repeatedly to decouple the same variables several times, to ensure higher connectivity for renormalization purposes. We foresee many other applications.

Although the present Letter is written in a boson field formalism, estimates for a potential theory situation with two- (or many-) body interactions, for example, are obtainable by considering terms developed herein by interpolation of the interaction alone (this is a trivial observation). The bounds we derive are proved by identifying the coefficients in a Taylor series, the method of proof given in [1]. This technique, not intimately tied to tree graphs, we view as more general than the tree graph oriented development in [4].

We consider the integral $E$

$$
E=\int \mathscr{D}_{\phi} \mathscr{N}(\mathscr{A}) \mathrm{e}^{-\mathscr{\infty}} \mathrm{e}^{-v} p
$$

where

$$
p=\not h \prod_{i \in 0} \phi_{i}
$$




$$
\begin{aligned}
& \mathscr{A}=\frac{1}{2} \sum \phi_{\imath} A_{\imath} \phi_{J}, \\
& V=\sum_{J} V_{J}, \\
& V_{J}=v_{J} \prod_{r \in J} \phi_{l}, \\
& \int \mathscr{D}_{\phi}=\prod_{l}\left(\int_{-\infty}^{\infty} \mathrm{d} \phi_{l}\right),
\end{aligned}
$$

and we introduce the covariance matrix $C$ inverse to $A$

$$
C_{t j}=\left(A^{-1}\right)_{t j}
$$

$\not 2, v_{J}$, and $A_{i}$ are numerical objects. The 'set' 0 in (2), and the 'sets' $J$ in (5), and other similar 'sets' to follow, are unordered and allow repetitions. (A 'set', such as 0 , is mathematically a mapping into the nonnegative integers, $i \rightarrow \alpha(i)=\alpha_{0}(i)$.) By $\Sigma_{i \in 0} f(i)$ we mean $\Sigma_{i} \alpha(i) f(i)$, and by $\Pi_{i \in 0} f(i)$ we mean $\Pi_{i}(f(i))^{\alpha(i)}$.) $\mathscr{N}(\mathscr{A})$ is a function of the $A_{y}$ chosen so that

$$
\int \mathscr{D}_{\phi} \mathscr{H}(\mathscr{A}) \mathrm{e}^{-\mathscr{P}}=1 .
$$

We use interpolation and integration by parts to write $E$ as a sum of terms

$$
E=\sum_{T} \int \mathrm{d} \sigma^{T} \int \mathscr{D}_{\phi} \cdot \mathcal{N}\left(\mathscr{A}^{T}\right) \mathrm{e}^{-\mathscr{A} T} \mathrm{e}^{-\nu T} I^{T},
$$

$\mathscr{A}^{T}$ is as $\mathscr{A}$ in (3), but with $C_{i j}$ replaced by $M_{i j}^{T} C_{y} . V^{T}$ is as $V$ in (4), but with $V_{J}$ replaced by $N_{J}^{T} V_{J} . M_{J J}^{T}$ and $N_{J}^{T}$ depend only on the parameters integrated over in $\int \mathrm{d} \sigma^{T} . I^{T}$ is a monomial in the $\phi$ 's. Expression (8) is generated inductively. We consider an expression (similar to a term in (8))

$$
\int \mathrm{d} \sigma^{t} \int \mathscr{D}_{\phi} \mathscr{N}(\mathscr{A}) \mathrm{e}^{-\mathscr{A}^{t}} \mathrm{e}^{-v^{t} I^{t}}
$$

Setting $\int \mathrm{d} \sigma^{t}=1, \mathscr{A}^{t}=\mathscr{A}, V^{t}=V, I^{t}=p$ we have our initial expression $E$. If we write $I^{t}=\phi_{\alpha} I^{t^{\prime}}$, then integration by parts replaces (9) with an integral with $\int \mathrm{d} \sigma^{t}$, $M_{t \jmath}^{t}, N_{J}^{t}$ unchanged, and $I^{t}$ replaced by a sum of terms

$$
\sum_{J} M_{\alpha, J}^{t} C_{\alpha, J} \mathrm{e}^{\nu t} \partial_{\phi_{j}}\left[I^{t^{\prime}} \mathrm{e}^{-\nu^{t}}\right]
$$

In total, (9) is replaced by a sum of terms of similar type.

In a single interpolation we introduce an interpolation parameter $s$ and functions $\alpha_{t y}(s), \beta_{J}(s)$ satisfying

$$
\alpha_{i}(1)=\beta_{J}(1)=1 .
$$


We let $f(s)$ be the expression in (9) with $M_{i j}^{t}$ replaced by $\alpha_{i j}(s) M_{i j}^{t}$ and $N_{J}^{t}$ replaced by $\beta_{J}(s) N_{J}^{t}$. Then, from the equality,

$$
f(1)=f(0)+\int_{0}^{\mathrm{1}} \mathrm{d} s \frac{\mathrm{d}}{\mathrm{d} s} f(s)
$$

We may replace (9) by a sum of terms of similar type. The first term on the right side of (12) yields a term similar to (9) with $M_{i j}^{t}$ replaced by $\alpha_{y}(0) M_{y j}^{t}$ and $N_{j}^{t}$ by $\beta_{J}(0) N_{J}^{t}$ and no other changes. From the last term in (12) there is also a sum of terms with replacements

$$
\int \mathrm{d} \sigma^{t} \rightarrow \int_{0}^{1} \mathrm{~d} s \int \mathrm{d} \sigma^{t}, \quad M_{i j}^{t} \rightarrow \alpha_{t j}(s) M_{y}^{t}, \quad N_{J}^{t} \rightarrow \beta_{J}(s) N_{J}^{t} .
$$

In (8), we may naturally write $I^{T}$ in the form

$$
I^{T}=\not\left(\prod_{J \in S_{J}^{T}}\left(-v_{J}\right)\right)\left(\prod_{(i, j) \in S_{C}^{T}} C_{i, J}\right) n^{T}(\sigma) \prod_{i \in S^{T}} \phi_{i} .
$$

$n^{T}(\sigma)$ contains all the dependence on interpolation parameters. We now set up an equivalence relation on the (terms labelled by) $T$ 's. We say $T_{1} \sim T_{2}$ if $S_{J}^{T_{1}}=S_{J}^{T_{2}}$ and $S_{C}^{T_{1}}=S_{C}^{T_{2}}$. That is, $T_{1} \sim T_{2}$ if they contain, 'differentiated down', the same interactions, $v_{J}$, and the same covariance elements, $C_{r, j}$, as exhibited in (14). In (14), and from now on, identifying $C_{i j}$ and $C_{j l}$, we consider only coefficients $C_{i, j}$ with $i \leqslant j$ for some ordering of the variables.

We assume the $\alpha$ 's and $\beta$ 's (as in (11)) satisfy (for $0 \leqslant s \leqslant 1$ )

$$
\alpha_{l j}(s) \geqslant 0, \quad \beta_{J}(s) \geqslant 0, \quad \alpha_{i j}^{\prime}(s) \geqslant 0, \quad \beta_{J}^{\prime}(s) \geqslant 0 .
$$

$\alpha_{\imath y}(s)$ is a symmetric matrix. We also assume sufficient positivity conditions allowing integrals to all exist, and to ensure absence of surface terms in integration by parts as defined in (9), (10).

We are now ready to present our basic combinatoric estimate. If $T_{0}$ is any term in the sum in (8) we have

$$
\sum_{T \sim T_{0}} \int \mathrm{d} \sigma^{T_{n}} n^{T}(\sigma) \leqslant \prod_{J \in R T_{0}} \frac{1}{\left(d_{J}^{\left.T_{0} !\right)}\right.} \prod_{J}\left(\frac{e_{J}^{T_{0} !}}{f_{J}^{T_{0} !}}\right),
$$

where (in the notation of (14))

(a) $R^{T_{0}}$ is the set of distinct $J$ in $S_{\mathrm{J}}^{T_{0}}$.

(b) $d_{J}^{T_{0}}$ is the number of times $J$ appears in $S_{J}^{T_{0}}$.

(c) $f_{J}^{T_{0}}$ is the number of times $j$ appears in $S^{T_{0}}$.

(d) $e_{j}^{T_{0}}=f_{j}^{T_{0}}+g_{j}^{T_{0}}$, where $g_{j}^{T_{0}}$ is the sum of the number of times $j$ appears as a left entry with the number of times it appears as a right entry in $S_{C}^{r_{0}}$.

The proof of (16) is similar to the proofs of (8.11) and (B.4) in [1]. We expand each term in (8) in a Taylor series in the $v$, and keep in each Taylor series only the single term in $\Pi_{J \in S_{J}}^{T_{0}} v_{J}$. 
We now do the integral over the $\phi_{i}$. (If the product over the $\phi_{i}$ in (14) contains an odd number of elements, we first multiply (8) by $\phi_{i_{0}}, i_{0}$ an arbitrary variable not in $I^{T_{0}}$.) We identify a particular product of covariances thus arising in term $T_{0}$ with nonzero coefficient. The conditions in (15) ensure this product arises with the same sign coefficient in all terms. The inequality (16) arises by identifying the coefficient of this product in each term in (8); the right side of (16) arises from the left side of (8).

The factor

$$
\prod_{j}\left(\frac{e_{j} !}{f_{J} !}\right)
$$

in (16) may not be the best bound obtainable by our proof. We have used the fact that if one extracts the coefficient of any particular monomial in the $C_{l j}$ from

$$
\int \mathscr{D}_{\phi} \mathscr{N}(\mathscr{A}) \mathrm{e}^{-\mathscr{A}}\left(\prod_{t}\left(\phi_{t}\right)^{e_{t}^{T_{0}}}\right)\left(\phi_{t_{0}}\right)^{\varepsilon},
$$

it is easily seen to be bounded by

$$
\prod_{j}\left(\frac{e^{T_{0}} !}{f_{j}^{T_{0} !}}\right)
$$

times the coefficient of the same monomial in the $C_{l j}$ from

$$
\int \mathscr{D}_{\phi} \mathscr{r}(\mathscr{A}) \mathrm{e}^{-\mathscr{A}}\left(\prod_{(i, J) \in S_{C}^{T_{0}}} C_{i, j}\right)\left(\prod_{\imath \in S^{T_{0}}} \phi_{\imath}\right)\left(\phi_{i_{0}}\right)^{\varepsilon} .
$$

$\varepsilon$ is 1 or 0 . We do not claim

$$
\prod_{j}\left(\frac{e_{j} !}{f_{j} !}\right)
$$

is the minimal such bound, for the particular monomial.

\section{References}

1. Battle, G. and Federbush, P., A phase cell cluster expansion for Euclidean field theories, Ann. Phys. 142, 95-139 (1982).

2. Battle, G. A. and Federbush, P., A note on cluster expansions, tree graph identities, extra $1 / N$ ! factors!!!, Lett. Math. Phys. 8, $55-57$ (1984).

3. Brydges, D. and Federbush, P., A new form of the Mayer expansion in classical statistical mechanics, J. Math. Phys. 19, 2064-2067 (1978).

4. Brydges, D., A short course on cluster expansions, Les Houches Summer School Notes (K. Osterwalder, ed.), 1984.

5. Glimm, J. and Jaffe. A., Quantum Physics, Springer-Verlag, Berlin. New York, 1981. 